\title{
Dante in Galizia: la nuova versione della Divina Comedia di Darío Xohán Cabana*
}

\author{
Benedict Buono \\ Universidade de Santiago de Compostela \\ ben.buono@usc.es
}

L'ostracismo di cui fu oggetto Dante nel primo Cinquecento, ampiamente alimentato dalla sua capacità di generare «un sistema straordinariamente complesso di forme e significati» ${ }^{1}$ - poco adatto al principo d'imitazione allora imperante ${ }^{2}$ - non impedì al poeta fiorentino di trasformarsi in un duraturo simbolo identitario, dal punto di vista letterario e linguistico, per generazioni di italiani, fossero essi letterati o lettori di più modeste pretese intellettuali. Era stato Pietro Bembo, incanalato sui binari di un esteriore decoro formale, a paragonarlo, nelle Prose della volgar lingua (2, XX), al loglio, la zizzania che soffoca le messi in un «bello e spazioso campo di grano»; 3 prima di lui, Giovan Francesco Fortunio, autore del primo trattato grammaticale della lingua italiana, le Regole grammaticali, non aveva risparmiato il suo biasimo per la licenza della lingua di Dante e la mancanza di rispetto verso la grammatica, ${ }^{4}$ pur essendo la Commedia il testo più citato all'interno dell'opera, ${ }^{5}$ situazione paradossale che ribadisce la centralità di questo nume tutelare dell'italiano lettera-

* Dante Alighieri, A Divina Comedia, ecc. A Coruña: Edicións da Curuxa, 2014.

1. Cfr. Pietro Trifone, Malalingua. Litaliano scorretto da Dante a oggi, Bologna: Il Mulino, 2011, p. 16.

2. Il pensiero non può che rivolgersi alle definizioni continiane di "plurilinguismo dantesco» e "unilinguismo petrarchesco» (Francesco Petrarca, Canzoniere, a cura di Gianfranco Contini, Torino: Einaudi, 1964, pp. X-XII), semplici etichette che, pur non esaurendo l'ampia gamma espressiva dei due autori, riassumono efficacemente il loro stile.

3. Giustificate dal contesto, d'altronde, Bembo si era guardato bene dal citare le espressioni «rozze e disonorate» di cui Dante si era servito «non senza biasimo» nei territori infernali, come «ed elli del cul aveva fatto trombetta»; «le mani alzò con amendue le fiche»; «'l tristo sacco che merda fa di quel che si trangugia»; «la puttana che rispuose al drudo suo» e altre simili, cfr. Pietro Trifone, Malalingua..., op. cit., p. 17.

4. In I 9, 10, 112; II 17, 29, 49 e 97, cfr. Gian Francesco Fortunio, Regole grammaticali della volgar lingua, a cura di Brian Richardson, Roma: Antenore, 2001, p. XIVIII.

5. In tutto 240 passi dell'Inferno, 97 del Purgatorio, 69 del Paradiso, accanto a cinque passi del Convivio, uno della Vita Nuova e quattro di canzoni delle Rime, rispetto ai 274 dei Rerum vulgarium fragmenta e ai 33 dei Trionfi, ivi, p. XLVII. 
rio. Lo stereotipo di Dante come «padre della lingua» — per alcuni studiosi un "pregiudizio [...] duro a morire ${ }^{6}$ —, non tradisce, dunque, una realtà di fatto: la preminenza di un personaggio avvertito come «icona di una comunità: non solo per la sua altezza poetica, ma per la capacità di far vibrare corde emotive largamente condivise», e la sua "ecumenicità», sia ai piani alti della lingua, nella tradizione poetica attraverso il dialogo intertestuale, sia ai piani bassi, con l'entrata in circolo nel linguaggio comune di espressioni memorabili o locuzioni desunte dalla Commedia. ${ }^{7}$ Non deve dunque sorprendere che confrontarsi con la cultura italiana, soprattutto per chi è straniero, voglia dire innanzitutto fare i conti con il magistero dantesco e con le diverse articolazioni plurilinguistiche offerte dalla Commedia, come attesta eloquentemente l'ultima traduzione in gallego di quest'opera, a cura del poeta Darío Xohán Cabana, nativo di A Coruña, edita, nel 2014, dalla casa editrice Edicións da Curuxa, versione aggiornata e perfezionata rispetto alla precedente, risalente al $1990 .{ }^{8}$ Possiamo ricavare maggiori informazioni dalle dichiarazioni dell'autore stesso nel prologo all'opera (Limiar, p. 23):

Esta de agora é completamente nova, resultado do traballo continuo e obsesivo destes tres últimos anos. Dos 14.233 versos que contén a Commedia, uns sete mil son agora diferentes na miña Comedia.

Con encomiabile rigore filologico, nell'introduzione, oltre a offrire una biografia del poeta fiorentino, volta soprattutto ad inserire l'opera nel quadro storico della Firenze due-trecentesca (Limiar pp. 11-20), Cabana indica le edizioni moderne su cui è stata esemplata la sua traduzione, senza dimenticare i commenti e le traduzioni in altre lingue. ${ }^{9}$ La sua nuova versione, con testo italiano a fronte, impreziosita dall'elegante veste tipografica, rinnova l'arduo

6. «[...] l'italiano sarebbe stato, per così dire, inventato quasi dal nulla ad opera di Dante, che lo avrebbe poi consegnato alle generazioni in forme perfette e immutabili. Un pregiudizio così duro a morire perché vivo soprattutto nella vulgata scolastica e alimentato dalla rinomanza internazionale che possiede a pieno diritto l'autore formidabile della Commedia", cfr, Riccardo Tesi, Storia dell'italiano. La formazione della lingua comune, Bologna, Zanichelli, 2007, p. 68.

7. Luca Serianni, «Echi danteschi nell'italiano letterario e non letterario», Italica, vol. 90, n. 2, 2013, pp. 291 e 296.

8. Dante Alighieri, A Divina Comedia, Nova traducción de Darío Xohán Cabana, A Coruña: Edicións da Curuxa, 2014 e ID., A Divina Comedia, Santiago de Compostela: Servizo Central de Publicacións da Xunta de Galicia, 1990, con illustrazioni di Xosé Luís De Dios e prologo di Giulia Lanciani.

9. Il testo seguito è quello dell'edizione Petrocchi, originariamente pubblicata in quattro volumi dalla Società Dantesca Italiana nel 1966-67, poi apparsa nella Piccola Biblioteca Einaudi nel 1975, a cui il traduttore ha apportato alcune modifiche, segnalate alle pp. 23-24 dell'introduzione. Accanto a questa si è avvalso delle edizioni di G. Vandelli (Milano, Hoepli, 1955); N. Sapegno (La Nuova Italia, Firenze, 1974); G. Giacalone (Signorelli, Roma, 1988); S. Jacomuzzi (SEI, Torino, 2008); G. Fallani (Newton, Roma, 2007 e 2013 (Limiar, pp. 23-24). Forse a un lettore più esperto sarebbe parsa opportuna una maggiore precisione nella segnalazione dei commenti e delle traduzioni consultate. 
proposito di tradurre le terzine dantesche, mantenendo endecasillabi e rime lungo tutta l'opera, ${ }^{10}$ operazione che ha costretto l'autore ad alcuni minuziosi e giustificati interventi lessicali, che illustreremo di seguito. In realtà - occorre ricordarlo_ l'instancabile attività di Xoán Cabana, nella sua veste di traduttore di classici italiani, aveva già avuto come frutto, negli anni scorsi, le due traduzioni dei Rerum vulgarium fragmenta di Petrarca, ${ }^{11}$ della Vita nuova di Dante, di un'antologia degli Stilnovisti e dei Fioretti di San Francesco, che sono state di certo una palestra poetica eccezionale per affrontare la complessità linguistica, stilistica, retorica, sintattica, rimica del principale testo dantesco, già paventata da Giulia Lanciani, autrice del prologo della prima versione della Commedia. ${ }^{12}$ Ma era possibile perfezionare l'edizione del 1990, grazie alla quale l'autore era già stato insignito, venticinque anni fa, della prestigiosa medaglia d'oro della città di Firenze, proprio il 25 maggio, genetliaco di Dante Alighieri? ${ }^{13}$ Sulla falsariga di Anxo Angueira, ${ }^{14}$ autore di un'entusiastica recensione di questa nuova versione della Divina Comedia di Cabana, si mettano a confronto i tre celeberrimi versi iniziali dell'opera: ${ }^{15}$

Originale: Nel mezzo del cammin di nostra vita / mi ritrovai per una selva oscura, I ché la diritta via era smarrita.

Edizione 1990: «No medio do camiño desta vida / vinme por unha selva tan escura / que a recta vía estaba confundida».

Edizione 2014: «No medio do camiño desta vida / acheime dentro dunha selva escura / que a recta vía fora confundida».

Le variazioni fondamentali riguardano il secondo verso. Innanzitutto, è da segnalare la maggior fedeltà prosodica al modello originale, grazie a dentro — che sostituisce unha —, sui cui cade, con maggiore intensità, l'accento in quarta dell'endecasillabo a minore. Più pertinente il ricorso al verbo acharse (esatto corrispondente del verbo ritrovarsi) rispetto a verse; in questo modo viene eliminato il tan della prima versione, a causa del quale il che iniziale del verso successivo acquisiva un valore causale, mentre nella seconda assume una valenza esplicativa. Il nuovo assetto permette la sostituzione di estaba confundida con fora confundida: lo smarrimento non dipenderebbe, così, esclusiva-

10. A volte, per motivi legati alla natura prosodica specifica della lingua d'arrivo, l'endecasillabo piano lascia spazio all'endecasillabo tronco (ad es. If XXV 1-2: ladro:squadro > ladrón: son), tipologia metrica del resto non sconosciuta a Dante.

11. La prima (Cancioneiro) è del 1989, la seconda, completa delle Rime disperse (Cancioneiro seguido das Rimas dispersas), del 2012; nel 1994 è uscita la Vida Nova; nel 2011 l'Antoloxía do Doce Estilo Novo; e, nel 2013, As Floriñas de San Francisco.

12. Darío Xohán Cabana, A Divina Comedia, op. cit., 1990, pp. VII-VIII.

13. Cfr. Limiar: pp. 22-23.

14. Cfr. Anxo Angueira, «A Divina Comedia de Dante Alighieri. Traducción de Darío Xohán Cabana", A Trabe de ouro, vol. XXV, t. IV, 2014, pp. 523-527.

15. Come nella traduzione di Cabana, uso il corsivo per l'originale italiano e il virgolettato per la trasposizione in gallego. 
mente dalla «retta via», ma assumerebbe un valore più intimo e personale. Ma veniamo a un altro esempio (IfIII, 91-93):

Originale: Ma poi che vide chio non mi partiva, / disse: «Per altra via, per altri porti / verrai a piaggia, non qui, per passare: / più lieve legno convien che ti porti». Versione 1990: «Pero despois, en vendo que eu non me iba, / Dixo: "Por outros portos e outra vía / debes ir, non por estes pra cruzar: / pra ti cómpre unha nave máis esguía”".

Versione 2014: «E logo dixo, vendo que eu non me iba: / "Por outros portos e por outra parte / tes que buscar a vía pra cruzar: / máis leve leño cómpre pra levarte"».

Nei primi due versi della nuova versione viene sacrificata l'espressione popolare en vendo que a favore di un più neutro vendo que, così da risparmiare due sillabe e anticipare il dixo (disse) del verso seguente, mantenendo la formulazione parallela (por outros, por outra) e la successione incrociata di suoni aspri presente nel secondo verso (outros - portos / outra parte); il nuovo sistema rimico favorisce l'eliminazione della nave della prima versione, recuperando, invece la metonimia leño e l'allitterazione leve leño del testo di riferimento: anche in questo caso la nuova traduzione si caratterizza per una maggiore fedeltà nei confronti dell'originale e una ricerca più approfondita dell'intreccio fonico-ritmico del verso dantesco.

Le novità non si limitano però alla sola traduzione, ma investono anche l'assetto paratestuale dell'opera, con l'aggiunta di utili note marginali di tipo storico-erudito a completamento dei dati forniti nell'introduzione, insieme ad altrettanto opportune annotazioni che chiariscono passi di particolare complessità interpretativa. ${ }^{16}$ Chiude il volume un repertorio di termini (Vocabulario ${ }^{17}$ di cui si è servito l'autore nel processo di traduzione, non registrati nel Diccionario de la Real Academia Galega, tuttavia nobilitati dal gallego medievale e dall'uso letterario successivo o sostenuti dall'uso comune. ${ }^{8}$ Ragioni metriche e stilistiche spingono infatti il traduttore a valersi di termini desueti o popolari, in linea, dunque con l'ampio registro lessicale dantesco, che mescidava sapientemente voci contemporanee, arcaiche e demotiche, sfruttando le possibilità di una lingua ricca nelle sue varietà diastratiche e diafasiche. ${ }^{19}$ Significativo, solo

16. Nella prima versione, le note, a pié di pagina, erano più che altro di tipo storico-erudito $\mathrm{e}$ non erano volte a giustificare particolari scelte di traduzione.

17. Darío Xohán Cabana, A Divina Comedia, op. cit., 2014, pp. 741-757.

18. Del resto, il ricorso a voci del gallego medievale, è usuale nel gallego moderno, cfr. Moisés Barreiro Comedeiro / Fátima Rodríguez Ruibal, «O recurso ó léxico medieval no galego moderno", in María Álvarez de la Granja / Ernesto Xosé González Seoane (a cura di), A estandarización do léxico, Santiago de Compostela: Consello da Cultura GalegaInstituto da Lingua Galega, 2003, pp. 349-385.

19. Pensiamo a voci prettamente 'basse' come bozzacchione 'susina deformata e gusta', parroffia 'parrocchia', piorno 'piovorno, pregno di vapore acqueo', manicare, introcque e altre simili, le ultime due esplicitamente condannate nel De vulgari eloquentia, cfr. Paola Manni, Il Trecento toscano, Bologna: Il Mulino, 2003, pp. 140-150. 
per citare qualche esempio, il ricorso al trisillabo pavura invece di pavor, non accolto dall'Accademia, eppure attestato nella poesia ottocentesca ("desde Pintos a Rosalía») o all'insieme letterario e popolare adentrarse («moi usado na fala e na literatura» glossa Cabana), afoularse ("popular e usado por Aquilino»), despertar («variante de espertar moi comun na lingua moderna, que xa se acha nas Cantigas de Santa Maria e na Cronica Troiana»), e via dicendo. Si tratta, del resto, di criteri non dissimili da quelli che avevano ispirato i redattori del Vocabolario della Crusca alla fine del Cinquecento, sulla falsariga del Salviati o del Varchi: la convergenza di fattori letterari e d'uso legittimava, infatti, l'accoglimento di una voce nel processo di spoglio, soprattutto laddove la lingua degli autori mostrava lacune lessicali. ${ }^{20}$ La serietà e la competenza con cui Cabana ha operato nel processo di traduzione emergono proprio da queste note in margine, a chiarimento di precise scelte anche a livello interpretativo, come nel caso della voce lonza, ${ }^{21}$ in cui si è preferito un prelievo dantesco all'originale pantera dell'edizione ' 90 , in ossequio a un principio di fedeltà testuale rispettosa del valore simbolico, scartando invece una semplice e passiva traduzione di tipo scientifico. ${ }^{22}$

Atteggiamento non dissimile è assunto nei confronti dei latinismi, che, pur presenti nel mondo infernale, si addensano gradatamente nell'ascesa ai territori celesti: ${ }^{23}$ si pensi a ineffabile - le cui quattro occorrenze sono localizzate nella seconda e terza cantica ( Pg XV 67, XXIX 29; Pd X 3, XXVII 7)—, mantenuto nella traduzione dei versi del Paradiso, ${ }^{24}$ proprio per la sua pregnanza teologica, mentre nei versi del Purgatorio se ne privilegia il valore semantico, sostituendolo con un sinonimo o fondendolo con il sostantivo a cui è riferito. ${ }^{25}$ Quando il latinismo, più che a privilegiare aspetti dottrinali, interviene a dare solennità allo stile, è assecondato, nella traduzione, dal ricor-

20. Queste coincidenze teoriche nella storia linguistica del gallego e dell'italiano emergono nella ripresa dell'espressione «questione della lingua» da parte di Francisco Fernadenz Rei a proposito del dibattito sulla normativa della lingua gallega a partire dagli anni Settanta del Novecento. Anche il gallego, a detta dello studioso, ha avuto, come l'italiano, le sue «tre corone»: Rosalía, Pondal e Curros, cfr. Francisco Fernández ReI, «A "questione della lingua" galega», Trabe de ouro, 2, t. 1, pp. 29-40.

21. If I, 32: una lonza leggiera e presta molto > «cunha lonza axilísima batín».

22. Così l'autore commenta la sua presa di posizione: "Calquera que sexa o animal real que designe, a palabra lonza non se deberá traducir por pantera -como habitualmente se fai, e como eu mesmo fixen hai vinte e cinco anos- pois aquí non estamos tratando de ciencias naturais senón de relixión e mito, e a pantera -sexa ela o que sexa- é simbolo de Cristo nos bestiarios medievais. [...] Todas as contas botadas, prefiro manter a palabra lonza, que mesmo ten a vantaxe de non existir en galego, en vez de intentar desentretiñar a auténtica natureza biolóxica dunha besta que aquí é estritamente simbólica», cfr. Darío Xohán CABANA, A Divina Comedia, op. cit., 2014, p. 34.

23. Cfr. Paola Manni, Il Trecento toscano, op. cit., pp. 151-53 e Vittorio Coletti, Storia dell'italiano letterario: dalle origini al Novecento, Torino: Einaudi, 1993, p. 48.

24. PdX 3: lo primo e ineffabile valore > "fixo o primeiro e inefable Valor"; XXVII 7: Oh gioia! oh ineffabile allegrezza! > "Ouh gozo! Ouh inefable alacridade!».

25. Pg XV 67: Quello infinito e ineffabil bene > "Aquel infindo e inexpresable ben»; XXIX 29: avrei quelle ineffabili delizie > "eu podería alí estas delicias». 
so a forme dotte (il corsivo è mio): ${ }^{26}$ si pensi al canto di Giustiniano ( $P d$ VI: «da li egregi romani» 43-44 > "os egrexios romanos»; "Quinzio, che dal cirro / negletto fu nomato" 46-47 > "Quincio, a quen o cirro / solto deu nome»; ${ }^{27}$ «là dov'Ettore si cuba» $68>$ "o lugar onde Héitor $c u b a » ; 28$ "la morte prese subitana e atra» $78>$ a morte [...] / súpeta e cruel ${ }^{29}$ ); "per l'omo in terra, se non fosse cive?» $P d$. VIII $116>$ «a vida do home se non fose cive?»; 30 «son di lor vero umbriferi prefazi» $P d$ XXX $78>$ «son da verdade umbriferos prefacios». ${ }^{31}$ Fra i tecnicismi di origine greca, investiti da sensi traslati, da segnalare l'aggettivo tetragono (PdXVII 24: ben tetragono ai colpi di ventura), mantenuto nella versione gallega («tetrágono prós golpes da ventura»). ${ }^{32}$ Lo stesso accade con la neoformazione teodia 'canto rivolto a Dio' ( $P d \mathrm{XXV} 73$ : 'Sperino in te', ne la sua tëodia) — costruito sulla base strutturale di comedia, melodìa, tragedia ${ }^{33}$ —, riprodotto fedelmente dal traduttore ("'Espera en ti', na súa teodía»). L'elenco potrebbe continuare, ciò che mi preme sottolineare è l'estrema attenzione del traduttore nei confronti dei termini dotti, che, nel primitivo uso dantesco, dovevano conferire all'enunciato un particolare stigma di eccezionalità, oggi in parte perduto, ${ }^{34}$ recuperato, in questa edizione, con la proposta di voci colte e desuete.

A detta degli studiosi, uno dei settori più innovativi e letterariamente produttivi della Commedia è rappresentato dai neologismi, e, fra questi, le formazioni parasintetiche, che —evidenzia Luca Serianni ${ }^{35}$ - hanno riscosso notevole successo nella tradizione poetica italiana. La maggior parte è costituita dai verbi parasintetici a prefisso in-, ricordiamo immegliarsi (PdXXX 87), incappellarsi (PdXXXII 72), incielare (Pd III 97), incinquarsi (Pd IX 40), indiarsi (Pd IV 28), indonnarsi (Pd VII 13), indovarsi (PdXXXIII 138), inforsarsi (Pd XXIV 87), infuturarsi (PdXVII 98), inluiarsi (Pd IX 73) e inleiarsi (PdXXII 127), inmillarsi (PdXXVIII 93), insemprarsi (PdX 148), insusarsi (PdXVII

26. Il corsivo è mio.

27. Nel Vocabulario: «Esta palabra usámola no sentido etimolóxico de crecha de pelo» (p. 743).

28. La nota a margine evidenzia che «Cuba é xurdio latinismo, cubat, xace» (p. 538).

29. Nel Vocabulario: «É tamén adxectivo, ben aboado na fala popular e na literatura, aínda que este uso non figure no DRAG. Pode significar repentino, ou aplicarse a algo con moita pendente $[\ldots]$ (p. 755).

30. Nota a margine: «O cru latinismo que tamén aparece en rima en Purg. XXXII 101».

31. Nota a margine: "Umbrifero é o latín umbrifer, sombrizo" (p. 710).

32. Attraverso l'uso figurato dantesco, il cui precedente è da individuare nel commento all'Etica aristotelica di san Tommaso, ha assunto il valore di 'solido, incrollabile' (Manni 154n), ribadito da Cabana in una nota specifica (p. 616).

33. Cfr. Paola Manni, Il Trecento toscano, op. cit., pp. 155-56.

34. E stato proprio l'uso dantesco, infatti, che ha permesso a molti latinismi danteschi di assumere una connotazione più comune e vulgata, cfr. Cfr. Paola Manni, Il Trecento toscano, op. cit., p. 153.

35. Cfr. Luca Serianni, Echi danteschi nellitaliano..., op. cit., pp. 295-296. Sempre sui verbi parasintetici danteschi, si vedano Riccardo TesI, Storia dellitaliano letterario..., op. cit., pp. 72-73; Paola Manni, Il Trecento toscano, op. cit., p. 159; e Vittorio Coletti, Storia dell'italiano letterario, op. cit., p. 51. 
13), intuarsi e inmiarsi (Pd IX 81), inurbarsi (Pg XXVI 69), inventrarsi (Pd XXI 84), inzaffirarsi (PdXXIII 102); con prefissi diversi: (a-) adduarsi (PdVII 6), appulcrare 'abbellire' (If VII 60), arruncigliare 'afferrare col ronciglio' (If XXI 75); (di-) dirocciarsi 'scendere giù dalla roccia' (IfXIV 115), dislagarsi 'elevarsi da una distesa d'acqua' ( $P g$ III 15), dismalare 'liberarsi dal male, purificare' (Pg XIII 3); (tras-) trasumanare 'trascendere l'umano' (Pd I 70), trasmodarsi 'oltrepassare ogni limite' (PdXXX 19). Qual è l'atteggiamento di Cabana nei loro confronti? Nella maggior parte dei casi presentati il traduttore opta per il mantenimento delle coniazioni dantesche, segnalandone l'unicità, nella nota a margine, in quanto "neoloxismo dantesco», con soluzioni in bilico fra il semplice adattamento e il calco-traduzione: abbiamo così encear, encincar, endearse, adonarse, enfuturarse, enventrarse, enmilarse, entuarse e enmiñarse, enelarse (per maschile e femminile), enurbarse, enzafirarse, apulcrar, deslagarse, desmalar, transhumanar; mentre nel caso di insemprarsi e indovarsi si ricorre a acotiarse, formato per l'appunto su cotio 'sempre', ed enondarse sul locativo onde. Altrove, per una maggiore trasparenza semantica, il traduttorre ricorre a delle parafrasi: simmegli («nos mellora»); s' incappelli ("faga [...] o seu capelo»); tinsusi ("cuxa altura fai»); sinforsa («non me inquieto»); arruncigliarmi («dar nos breus»); si diroccia («se enfreita»); e trasmoda («da usanza [...] trascende»). Anche in questo caso Cabana preferisce attenersi alla compagine lessicale dell'antigrafo, consapevole dell'importanza del neologismo in quanto fonte di arricchimento lessicale ed espressione della potente creatività dantesca. Procedimento analogo si riscontra nella traduzione dei nomi del drappello di diavoli in cui s'imbatte Dante in If XXI: da una parte abbiamo Alichino (Aliquino); Barbariccia (Barbariza); Draghignazzo (Draguñazo); Rubicante (Rubicante); dall'altra Malebranche (Malaspoutas < mala + pouta 'zampa'); Malacoda (Rabomau < rabo 'coda' + mal); Scarmiglione (Esguedellón, formato sul verbo esguedellar 'scarmigliare'); Calcabrina (Tripaorballo, da tripar 'calcare' + orballo 'brina'); Cagnazzo (Canxigante); Libicocco (in cui l'incrocio fra i nomi dei due venti libeccio e scirocco è reso con Avregón, da ávrego, 'vento del sud o sud-ovest'); Cirïatto (Quiro 'porco'); Graffiacane (Campouta, da can + pouta visto precedentemente); e Farfarello (Trasno, da trasno, 'spiritello notturno dispettoso e vivace'). ${ }^{36}$ La traduzione di questa serie di nomi asseconda pienamente la creatività dantesca, sulla base di termini comuni opportunamente modificati per acquistare un significato allusivo alle qualità fisiche o caratteriali dei vari personaggi demoniaci, preservando «l'elemento di tracotanza e di aggressività da farsa, quale appunto Dante aveva in mente nel comporre que-

36. E' Cabana stesso ad avvertire il lettore, in una nota a margine, di questa scelta traduttiva: «case todos os nomes destes demos teñen sensos transparantes, e por iso preferimos reconstruílos na traducción, en canto de adapatálos coma de costume», cfr. Darío Xohán Cabana, A Divina Comedia, op. cit., 2014, p. 164. Sul significato dei nomi dei diavoli, cfr. Dante Alighieri, Commedia, con il commento di Anna Maria Chiavacci Leonardi, Milano: Arnoldo Mondadori, 1991-97, I vol., p. 650. 
sto interludio", dove l'elemento visivo si fonde con quello fonico. ${ }^{37}$ Elemento fonico, determinante nella descrizione delle lande infernali, come nella rappresentazione del paesaggio stravolto del canto di Pier delle Vigne (If XIII, 1-30), vero trionfo della negatività, rovesciamento delle immagini tradizionali del locus amoenus: vi si addensano le rime consonantiche aspre in -osco, -olti, -entre, -onchi ed -erpi, a volte paronomastiche (bronchi : tronchi; pruno : bruno; scerpi : serpi : sterpi), che culminano nel polittoto Cred'io ch'ei credette ch'io credessi (v. 25), minuziosamente riprodotte anche nella traduzione (espesura: escura : agrura I tortas : hortas: retortas / ventres : adentres : namentres / gallas : tallas : barallas), accanto al verso finale "Creo que el creu que eu creía que viñese».

Nella sua recensione alla Divina Comedia di Darío Xohán Cabana, Anxo Angueira ${ }^{38}$ ricorda il forte legame esistente fra Dante e la Galizia, attestato prima nella Vita Nova (XLV), ${ }^{39}$ poi nel XXV canto del Paradiso, quando Beatrice indica al protagonista il lume di San Giacomo Maggiore: «e la mia donna, piena di letizia, / mi disse: «Mira, mira: ecco il barone / per cui là giù si vicita Galizia» (vv. 16-18). L'ammirazione per il poeta fiorentino in questa regione della Spagna emerge dallo studio del fondo storico della Biblioteca Xeral dell'Università di Santiago, che custodisce un magnifico esemplare dell'incunabolo della Commedia, stampato a Firenze nel 1481 per i tipi di «Nicholo di Lorenzo della Magna", con il commento dell'umanista fiorentino Cristoforo Landino ${ }^{40}$, insieme alla prima versione cinquecentesca in castigliano dell'opera dantesca, curata da Pedro Fernández de Villegas: ${ }^{41}$ a ragione, dunque, potremo inserire l'accurata traduzione di Xohán Cabana nel solco di questa secolare tradizione di scambi culturali fra l'Italia e la Galizia, di cui il libro è stato - e continua ad essere- l'indiscusso protagonista. ${ }^{42}$

37. Ivi, p. 647.

38. Cfr. Anxo Angueira, «A Divina Comedia de Dante Alighieri..., op. cit., p. 527.

39. «E però da sapere che in tre modi si chiamano propriamente le genti che vanno al servigio de l'Altissimo: chiamansi palmieri in quanto vanno oltremare, la onde molte volte recano la palma; chiamansi peregrini in quanto vanno a la casa di Galizia, però che la sepoltura di Sa' Iacopo fue più lontana della sua patria che d'alcuno altro apostolo; chiamansi romei quanti vanno a Roma».

40. Segnatura Res 19818. La Biblioteca conserva un altro esemplare della Commedia commentata dal Landino, edita "Appresso Giouambattista Marchio Sessa, \& fratelli» nel 1578 (segnatura 4846).

41. Segnatura 12562. Si tratta di un’opera rara: «Según el inventario que nos ofrecen Alvar y Lucía Megías (2009: 298), se tienen noticias de 45 ejemplares de este impreso, que se hallan esparcidos en diferentes bibliotecas europeas y norteamericanas", cfr. Cinthia M. Hamlin, "La transmisión textual de la traducción de la Divina Comedia (1515): ¿ del impreso al manuscrito?», Revista de Filología Española, XCIII, n. 2, 2013, pp. 273-289; la citazione è tratta da pag. 273.

42. Per la storia del fondo italiano antico della biblioteca universitaria di Santiago, mi permetto di rinviare a Benedict BuONo, "La ricezione della cultura italiana del Rinascimento in Spagna: il fondo italiano antico della Biblioteca Universitaria di Santiago de Compostela», Rivista di letteratura italiana, 2011, n. 1, pp. 101-111. 